\title{
Analysis and Design of Thermohydrodynamic on the Hydraulic Servo Cylinder*
}

\author{
Rong-Tai YANG ${ }^{* *}$, Yung-Kuang YANG**, Shieu-Shien LIN $^{* * *}$ and Ming-Chang JENG ${ }^{* *}$ \\ In this study, analysis and design of the hydraulic servo cylinder by using dual conical- \\ cylindrical bearing with practical dimension is considered, which can be expressed by di- \\ mensionless forms for bearing performance parameters. The film viscosity is formulated \\ as an exponential function of temperature and pressure. The thermohydrodynamic bound- \\ ary condition is the basic assumptions of this analysis. Also, effect of the eccentricity ratio, \\ misalignment factor and film thickness are considered in herein.
}

Key Words: Dual Conical-Cylindrical Bearing, Viscosity-Pressure-Temperature Relationships, Thermohydrodynamic

\section{Introduction}

The structure of conical bearing is modified herein to be single conical-cylindrical configuration known as a taper-land slider bearing. The dual bearing is designed symmetrically such that the halves are opposite single conical-cylindrical, and the oil pressure is applied to the middle port of the bearing. The dual conical-cylindrical bearing is dealt with using the electro-hydraulic servo cylinder, which offers a major mechanism for improving the control accuracy, eliminating the static friction, and increasing the response of dynamics and the normal load carrying capacity for the electro-hydraulic servo systems.

Yang and Jeng using dimensionless variables had studied the progression in conical-cylindrical bearing. Such as in Ref.(1), examined the effect of eccentricity and misalignment on the performance parameters of the dual conical-cylindrical bearing with thermal effects under constant supply pressure. Which found that the normal load carrying capacity is less relevant to velocity under the same conditions of eccentricity ratio, misalignment factor and temperature boundary. In Ref. (2), compared the thermal effects on single conical-cylindrical bearing between 2-D and 3-D energy equations. The calculation results reveal that, the difference in bearing perfor-

\footnotetext{
* Received 22nd March, 2004 (No. 04-5035)

** Department of Mechanical Engineering, Ming Hsin University of Science and Technology, 1 Hsin Hsing Road, Hsin Feng, 304 Hsinchu, Taiwan. E-mail: yky@must.edu.tw

*** Department of Mechanical Engineering, National Central University, Chung-Li 32054, Taiwan
}

mance between 2-D and 3-D energy equations is not significant. In Ref. (3), studied further examines thermohydrodynamic analysis of the single conical-cylindrical bearing. A power-law fluid model is used, in which the thermohydrodynamic boundary condition is considered. In Ref.(4), the effects of temperature and pressure on the dual conical-cylindrical bearing using 3-D energy equations with Newtonian lubricants inspected. The viscositypressure-temperature variations are considered; because of the supply pressure is very high $(21 \mathrm{MPa})$. The results reveal that pressure increases both the film viscosity and temperature.

In this study, further analysis and design of the hydraulic servo cylinder by using dual conical-cylindrical bearing is considered. For instance, the electro-hydraulic servo cylinder is used in damper test system equipments which high speed is needed. The damper test system applied on functional evaluation of ground vehicle damper performance that can be included friction force testing. In this study, specifications of the electro-hydraulic servo cylinder are: piston rod diameter $45 \mathrm{~mm}$, piston effective area $13.55 \mathrm{~cm}^{2}$, dynamic force $25 \mathrm{kN}$ (i.e., $21 \mathrm{MPa}$ supply pressure), no load maximum velocity $4 \mathrm{~m} / \mathrm{s}$ (i.e., servovalve rated flow 340 liter $/ \mathrm{min}$ ).

\section{Nomenclature}

$c_{0}$ : specific heat of lubricant, $(\mathrm{J} / \mathrm{kgK})$

$d$ : cylinder rod diameters, $(\mathrm{m})$

$\bar{e}$ : eccentricity ratio at one end, $\bar{x}=0,\left(e / h_{0}\right)$

$f$ : tangential (friction) force, $(\mathrm{N})$

$\bar{f}$ : dimensionless shear force

$h:$ film thickness, (m) 
$\bar{h}$ : dimensionless film thickness

$h_{c}$ : convective heat transfer coefficient, $\left(\mathrm{W} / \mathrm{m}^{2} \mathrm{~K}\right)$

$h_{0}$ : outlet film thickness, (m)

$\bar{h}_{0}$ : dimensionless outlet film thickness

$k_{0}$ : thermal conductivity of lubricant, $(\mathrm{W} / \mathrm{mK})$

$k_{p}$ : thermal conductivity of stationary component, $(\mathrm{W} / \mathrm{mK})$

$L:$ length-diameter ratio, $(l / d)$

$l$ : length in $x$ direction, (m)

$n_{s}:$ shoulder length ratio

$p$ : pressure, $\mathrm{Pa}$ (or $\mathrm{N} / \mathrm{m}^{2}$ )

$\bar{P}$ : dimensionless pressure

$\bar{p}_{s}:$ dimensionless supply pressure

$r:$ cylinder rod radius, $(\mathrm{m})$

$s_{h}:$ shoulder height, $(\mathrm{m})$

$T$ : film temperature, $\left({ }^{\circ} \mathrm{C}\right)$

$T_{a}$ : ambient temperature, $\left({ }^{\circ} \mathrm{C}\right)$

$T_{\text {in }}$ : lubricant temperature supplied, $\left({ }^{\circ} \mathrm{C}\right)$

$T_{p}$ : temperature in stationary component, $\left({ }^{\circ} \mathrm{C}\right)$

$t_{p}$ : thickness of stationary component, (m)

$u$ : film velocity in $x$ direction, $(\mathrm{m} / \mathrm{s})$

$\bar{u}$ : dimensionless film velocity in $x$ direction

$u_{b}:$ surface $b$ (or cylinder rod) velocity, $(\mathrm{m} / \mathrm{s}$ )

$v:$ film velocity in $y$ direction, $(\mathrm{m} / \mathrm{s})$

$\bar{v}:$ dimensionless film velocity in $y$ direction

$w:$ film velocity in $z$ direction, $(\mathrm{m} / \mathrm{s})$

$\bar{w}:$ dimensionless film velocity in $z$ direction

$w_{z}$ : normal load carrying capacity, $(\mathrm{N})$

$\bar{w}_{z}$ : dimensionless normal load carrying capacity

$x$ : coordinate system in the moving direction used in lubricant, (m)

$x_{p}$ : coordinate system used in stationary component, (m)

$y$ : coordinate system in circumferential direction, (m)

$z$ : coordinate system across the film used in lubricant, (m)

$z_{p}$ : coordinate system used in stationary component, (m)

$\alpha$ : pressure-viscosity coefficient, $\left(\mathrm{m}^{2} / \mathrm{N}\right)$

$\bar{\alpha}:$ dimensionless pressure-viscosity

$\beta$ : temperature-viscosity coefficient, $(1 / \mathrm{K})$

$\gamma_{1}, \gamma_{2}:$ tilt angles, (degree)

$\eta:$ absolute viscosity, $\mathrm{Pa}$ (or $\mathrm{N} / \mathrm{m}^{2}$ )

$\eta_{0}$ : absolute viscosity at $p=0$ and constant temperature, $\mathrm{Pa} \cdot \mathrm{s}\left(\right.$ or $\left.\mathrm{N} \cdot \mathrm{s} / \mathrm{m}^{2}\right)$

$\bar{\eta}$ : dimensionless absolute viscosity

$\bar{\theta}$ : dimensionless circumferential angle

$\mu:$ frictional coefficient

$\bar{\mu}$ : frictional number

$\rho_{0}:$ density of lubricant, $\left(\mathrm{kg} / \mathrm{m}^{3}\right)$

$\sigma_{1}, \sigma_{2}$ : two independent misalignment factor

$\phi$ : circumferential angle, $(y / r)$

\section{Schematic of the Hydraulic Servo Cylinder}

Figure 1 shows the mechanisms studied herein for the dual conical-cylindrical configuration. Each bearing has been designed symmetrically, the halves having opposite tapers and lands. The piston rod moves to the right and the oil inside the left and right-sided plenum flows back to the reservoir when the oil pressure is applied to the middle port of the bearing. The bearing oil film thickness varies along the axial and circumferential directions depending on the geometric configuration of the bearing and the effects of shoulder length ratio, eccentricity ratio, and tilt angles.

The dual conical-cylindrical bearing is dealt with using the electro-hydraulic servo cylinder as shown in Fig. 2, which offers a major mechanism for improving the control accuracy, eliminating the static friction, and increasing the response of dynamics and the normal load carrying capacity for the electro-hydraulic servo systems.

The mechanism on the left-sided of Fig. 1 is an extension of the taper-land slider bearing. The minimum film thickness is $h_{0}$ with a length of $0 \leq x \leq l\left(1-n_{s}\right) / 2$, and the film thickness increases linearly from the minimum film

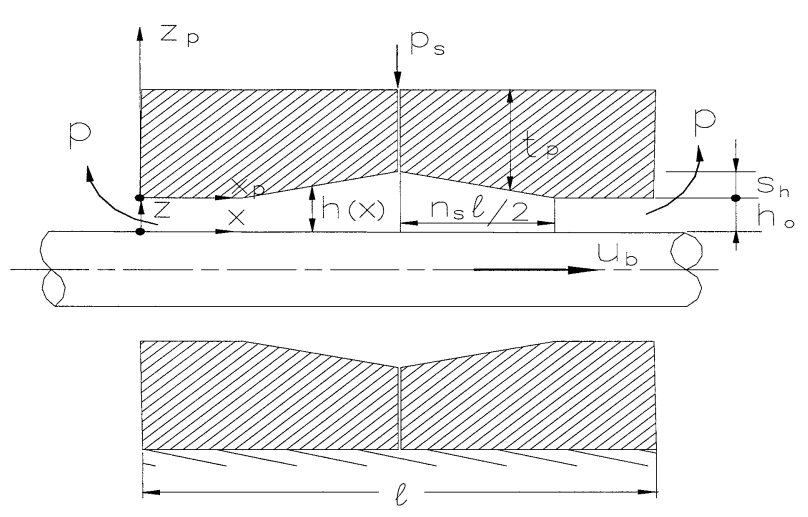

Fig. 1 Dual conical-cylindrical bearing configuration ${ }^{(4)}$

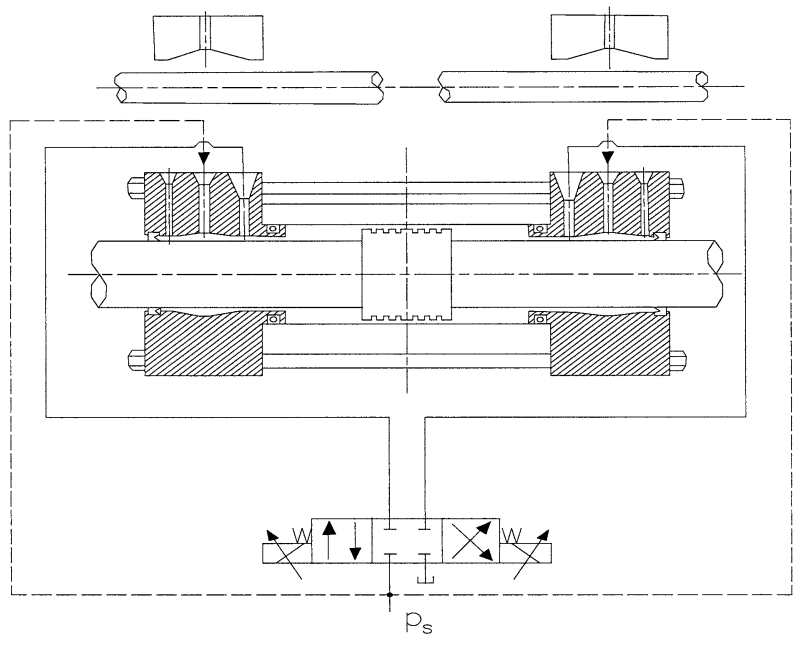

Fig. 2 Electro-hydraulic servo cylinder configuration ${ }^{(2)}$ 


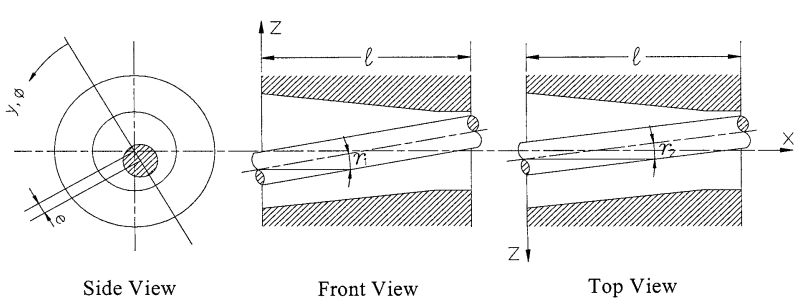

Fig. 3 The geometry of eccentric and asymmetric ${ }^{(2)}$

thickness $h_{0}$ to $\left(h_{0}+s_{h}\right)$ with a length of $l\left(1-n_{s}\right) / 2 \leq x \leq$ $l / 2$, and decreases linearly from $\left(h_{0}+s_{h}\right)$ to $\left(h_{0}\right)$ with a length of $l / 2 \leq x \leq l\left(1+n_{s}\right) / 2$. The minimum film thickness is $h_{0}$ with a length of $l\left(1+n_{s}\right) / 2 \leq x \leq l$ on the right-sided bearing.

The geometry of eccentric and asymmetric film thickness is illustrated in Fig. 3, where $e$ is eccentricity ratio, $\gamma_{1}$ and $\gamma_{2}$ are the tilt angles, and $x=0$ is the reference point at the leftmost side of the bearing. Buckholz and Lin $^{(5)}$ and Jang and Chang ${ }^{(6)}$ also depicted the film thickness geometry of the journal bearing in a similar manner.

The following transformations were used to nondimensionalize the governing equation:

$$
\begin{aligned}
& \bar{x}=\frac{x}{l}, \quad \bar{x}_{p}=\frac{x_{p}}{l}, \quad L=\frac{l}{d}, \quad \bar{h}_{0}=\frac{h_{0}}{s_{h}}, \quad \bar{h}=\frac{h}{s_{h}}, \\
& \bar{l}=\frac{l}{s_{h}}, \quad \bar{t}_{p}=\frac{t_{p}}{s_{h}}, \quad \bar{u}=\frac{u}{u_{b}}, \quad \bar{w}=\frac{l w}{s_{h} u_{b}}, \quad \bar{v}=\frac{v}{u_{b}}, \\
& \bar{z}=\frac{z}{h}, \quad \bar{z}_{p}=\frac{z_{p}}{t_{p}}, \quad \bar{p}=\frac{p s_{h}^{2}}{\eta_{0} u_{b} l}, \quad \bar{w}_{z}=\frac{w_{z} s_{h}^{2}}{\eta_{0} u_{b} d l^{2}}, \\
& \bar{q}_{x}=\frac{q_{x}}{u_{b} s_{h} d}, \quad \bar{f}=\frac{f s_{h}}{\eta_{0} u_{b} d l}, \quad \bar{T}=\frac{T}{T_{i n}}, \quad \bar{T}_{p}=\frac{T_{p}}{T_{i n}}, \\
& \bar{T}_{a}=\frac{T_{a}}{T_{i n}}, \quad \bar{\beta}=\beta T_{i n}, \quad \bar{\theta}=\frac{\phi}{\pi}=\frac{2 y}{\pi d}, \quad \bar{\eta}=\frac{\eta}{\eta_{0}}, \quad \bar{\mu}=\frac{\mu l}{s_{h}}
\end{aligned}
$$

The equation of film thickness in dimensionless form can be expressed as:

$$
\bar{h}=\left\{\begin{array}{c}
\bar{h}_{0}(1+\bar{e} \cos \pi \bar{\theta})-\bar{x} \sigma_{1} \cos \pi \bar{\theta}+\bar{x} \sigma_{2} \sin \pi \bar{\theta} \\
\text { at } 0 \leq \bar{x} \leq \frac{\left(1-n_{s}\right)}{2}, \bar{p}(0, \bar{\theta})=0 \\
\bar{h}_{0}(1+\bar{e} \cos \pi \bar{\theta})+\left[\frac{\bar{x}-\left(1-n_{s}\right) / 2}{n_{s} / 2}\right] \\
-\bar{x} \sigma_{1} \cos \pi \bar{\theta}+\bar{x} \sigma_{2} \sin \pi \bar{\theta} ; \\
\text { at } \frac{\left(1-n_{s}\right)}{2} \leq \bar{x} \leq \frac{1}{2}, \bar{p}\left(\frac{1}{2}, \bar{\theta}\right)=\bar{p}_{s} \\
\bar{h}_{0}(1+\bar{e} \cos \pi \bar{\theta})+\left[\frac{\left(1+n_{s}\right) / 2-\bar{x}}{n_{s} / 2}\right] \\
-\bar{x} \sigma_{1} \cos \pi \bar{\theta}+\bar{x} \sigma_{2} \sin \pi \bar{\theta} ; \\
\text { at } \frac{1}{2} \leq \bar{x} \leq \frac{\left(1+n_{s}\right)}{2}, \bar{p}\left(\frac{1}{2}, \bar{\theta}\right)=\bar{p}_{s} \\
\bar{h}_{0}(1+\bar{e} \cos \pi \bar{\theta})-\bar{x} \sigma_{1} \cos \pi \bar{\theta}+\bar{x} \sigma_{2} \sin \pi \bar{\theta} ; \\
\text { at } \frac{\left(1+n_{s}\right)}{2} \leq \bar{x} \leq 1, \bar{p}(1, \bar{\theta})=0
\end{array}\right.
$$

where $\bar{x}=\frac{x}{l}, \bar{e}=\frac{e}{h_{0}}, \bar{h}_{0}=\frac{h_{0}}{s_{h}}, \sigma_{1}=2\left(r / s_{h}\right)(l / d) \tan \gamma_{1}, \sigma_{2}=$ $2\left(r / s_{h}\right)(l / d) \tan \gamma_{2}$, (e.g., $\left.\tan \gamma_{1} \approx \gamma_{1}, \tan \gamma_{2} \approx \gamma_{2}\right)$

It is noted that the geometry is referenced to the eccentricity at $\bar{x}=0$. Two independent misalignment angles, $\sigma_{1}$ and $\sigma_{2}$, are measured from $\bar{x}=0$ and the film thickness geometry depends upon $\bar{x}$ and $\bar{\theta}$.

\section{Governing Equations}

\subsection{Reynolds equation}

The configuration in Fig. 1 can be treated as a slider bearing; therefore, the two-dimensional Reynolds equation can be expressed as:

$$
\frac{\partial}{\partial \bar{x}}\left(\frac{\bar{h}^{3}}{\bar{\eta}} \frac{\partial \bar{p}}{\partial \bar{x}}\right)+\frac{\partial}{\partial \bar{y}}\left(\frac{\bar{h}^{3}}{\bar{\eta}} \frac{\partial \bar{p}}{\partial \bar{y}}\right)=6 \frac{\partial \bar{h}}{\partial \bar{x}}
$$

Equation (2.a) can be converted into conical-cylindrical bearing model. Then the two-dimensional Reynolds equation can be expressed as:

$$
\frac{\partial}{\partial \bar{x}}\left(\frac{\bar{h}^{3}}{\bar{\eta}} \frac{\partial \bar{p}}{\partial \bar{x}}\right)+\left(\frac{L}{\pi / 2}\right)^{2} \frac{\partial}{\partial \bar{\theta}}\left(\frac{\bar{h}^{3}}{\bar{\eta}} \frac{\bar{\partial} \bar{p}}{\partial \bar{\theta}}\right)=6 \frac{\partial \bar{h}}{\partial \bar{x}}
$$

The pressure boundary conditions in dimensionless form are:

$$
\begin{aligned}
& \bar{p}(0, \bar{\theta})=\bar{p}(1, \bar{\theta})=0 \quad \text { (in motion direction) } \\
& \bar{p}(\bar{x}, 0)=\bar{p}(\bar{x}, 2) \quad(\text { in the circumferential direction) } \\
& \bar{p}\left(\frac{1}{2}, \bar{\theta}\right)=\bar{p}_{s} \quad \text { (in the middle port of supply pressure) }
\end{aligned}
$$

In addition, the pressure at the supply oil port is taken to be equal to the supply oil pressure, measured relative to the ambient one. Since cavitation is not considered, the pressure is constrained to be positive throughout the oil film. At the point of film rupture to the left of the bearing, the following Reynolds pressure boundary conditions must be applied:

$$
\bar{p}(\bar{x}, \bar{\theta})=\frac{\partial \bar{p}}{\partial \bar{x}}=0
$$

\subsection{Oil film velocity profiles}

The piston rod does not rotate and motion in the direction of film thickness. Therefore, the direction of circumference film velocity $(\bar{v})$ and the direction of thickness film velocity $(\bar{w})$ are far less than film velocity $(\bar{u})$ of motion direction. The oil film velocity profiles along $x, y$ and $z$-directions can be expressed as:

$$
\begin{aligned}
\bar{u} & =1-\frac{\bar{z}}{\bar{h}}+\frac{\bar{z}^{2}}{2 \bar{\eta}} \frac{d \bar{p}}{d \bar{x}}-\frac{\bar{z} \bar{h}}{2 \bar{\eta}} \frac{d \bar{p}}{d \bar{x}} \\
\bar{v} & =\left(\frac{\bar{z}^{2}}{2 \bar{\eta}}-\frac{\bar{z} \bar{h}}{2 \bar{\eta}}\right)\left[\left(\frac{L}{\pi / 2}\right) \frac{d \bar{p}}{d \bar{\theta}}\right] \\
\bar{w} & =\left(\frac{s_{h}}{l}\right)\left\{-\left(\frac{\bar{z}^{3}}{6 \bar{\eta}}-\frac{\bar{h} \bar{z}^{2}}{4 \bar{\eta}}\right)\left[\frac{d^{2} \bar{p}}{d \bar{x}^{2}}+\left(\frac{L}{\pi / 2}\right)^{2} \frac{d^{2} \bar{p}}{d \bar{\theta}^{2}}\right]\right. \\
& +\frac{\partial}{\partial \bar{x}}\left(\frac{\bar{z}^{2}}{2 \bar{h}}\right)-\frac{\bar{z}}{\bar{h}} \frac{\partial \bar{h}}{\partial \bar{x}} \frac{\partial}{\partial \bar{z}}\left(\frac{\bar{z}^{2}}{2 \bar{h}}\right)-\frac{\partial}{\partial \bar{x}}\left(\frac{\bar{z}^{3}}{6 \bar{\eta}}-\frac{\bar{h} \bar{z}^{2}}{4 \bar{\eta}}\right) \frac{d \bar{p}}{d \bar{x}}
\end{aligned}
$$




$$
\begin{aligned}
& +\frac{\bar{z}}{\bar{h}} \frac{\partial \bar{h}}{\partial \bar{x}} \frac{\partial}{\partial \bar{z}}\left(\frac{\bar{z}^{3}}{6 \bar{\eta}}-\frac{\bar{h} \bar{z}^{2}}{4 \bar{\eta}}\right) \frac{d \bar{p}}{d \bar{x}} \\
& \left.-\left(\frac{L}{\pi / 2}\right)^{2} \frac{\partial}{\partial \bar{\theta}}\left(\frac{\bar{z}^{2}}{6 \bar{\eta}}-\frac{\bar{h} \bar{z}^{2}}{4 \bar{\eta}}\right) \frac{d \bar{p}}{d \bar{\theta}}\right\}
\end{aligned}
$$

From Eq. (3), we know that three-direction velocity distribution and viscosity were related, and the viscosity can be denoted as the exponential function of film temperature and pressure according to as in Ref. (3). Also the variation of film temperature depended on thermohydrodynamics boundary condition.

\subsection{Energy equation}

4.3.1 Energy equation in the oil film A steady state three-dimensional energy equation as in reference Ezzat and Rohde ${ }^{(7)}$, and Jang and Khonsari ${ }^{(8)}$ expressing a point-wise balance of convective and conductive heat transfer with viscous dissipation in the oil film can be expressed as:

$$
\begin{gathered}
\bar{u}\left(\frac{\partial \bar{T}}{\partial \bar{x}}-\frac{\bar{z}}{\bar{h}} \frac{\partial \bar{h}}{\partial \bar{x}} \frac{\partial \bar{T}}{\partial \bar{z}}\right)+\bar{v}\left(\frac{L}{\pi / 2}\right)\left(\frac{\partial \bar{T}}{\partial \bar{\theta}}\right)+\bar{w}\left(\frac{l}{s_{h}}\right)\left(\frac{\partial \bar{T}}{\partial \bar{z}}\right) \\
=\frac{\lambda_{2}}{\lambda_{1}} \frac{\partial^{2} \bar{T}}{\partial \bar{z}^{2}}+\frac{\lambda_{3}}{\lambda_{1}} \bar{\eta}(\bar{T})\left[\left(\frac{\partial \bar{u}}{\partial \bar{z}}\right)^{2}+\left(\frac{\partial \bar{v}}{\partial \bar{z}}\right)^{2}+\left(\frac{\partial \bar{w}}{\partial \bar{z}}\right)^{2}\right]
\end{gathered}
$$

where: $\lambda_{1}=\frac{\rho_{0} c_{0} l u_{b}}{k_{0}}, \lambda_{2}=\left(\frac{l}{s_{h}}\right)^{2}, \lambda_{3}=\left(\frac{l}{s_{h}}\right)^{2} \frac{\eta_{0} u_{b}^{2}}{k_{0} T_{\text {in }}}$

The terms on the Left-Hand-Side represent the energy transported by convection. The first terms on the RightHand-Side represent the heat transfer by conduction and the last term on the R.H.S. represents the energy generated by internal friction known as viscous dissipation term.

The boundary conditions used for Eq. (4) are:

$$
\begin{array}{lll}
\bar{T}=1 & \text { at } & \bar{x}=0 \\
\bar{T}=1 & \text { at } & \bar{z}=0
\end{array}
$$

In addition, the thermal boundary conditions at the oil-solid interface for the thermohydrodynamic (THD) solution conditions studied can be expressed as:

$$
\frac{\partial \bar{T}}{\partial \bar{z}}=\left.\bar{h} \frac{k_{p}}{k_{0}} \frac{s_{h}}{t_{p}} \frac{\partial \bar{T}_{p}}{\partial \bar{z}_{P}}\right|_{\bar{z}_{p}=0} \quad \text { at } \quad \bar{z}=1
$$

where: $\bar{T}_{p}=\frac{T_{p}}{T_{\text {in }}}, \bar{z}_{p}=\frac{z_{p}}{t_{p}}$

\subsubsection{Energy equation in the stationary solid} The differential equation governing the heat transfer in the bearing stationary component is Laplace heat-conduction equation, which is coupled to Eq. (4) through the boundary conditions Eqs. (5) and (6). The two-dimensional in dimensionless form of heat-conduction equation governing the stationary pad can be expressed as:

$$
\frac{\partial^{2} \bar{T}_{p}}{\partial \bar{x}_{p}^{2}}+\left(\frac{l}{t_{p}}\right)^{2} \frac{\partial^{2} \bar{T}_{p}}{\partial \bar{z}_{p}^{2}}=0
$$

where: $\bar{x}_{p}=\frac{x_{p}}{l}$
The boundary conditions in dimensionless form for the heat conduction equation are:

$$
\begin{aligned}
& \frac{\partial \bar{T}_{p}}{\partial \bar{z}_{p}}=\left.\frac{1}{\bar{h}} \frac{k_{0}}{k_{p}} \frac{t_{p}}{s_{h}} \frac{\partial \bar{T}}{\partial \bar{z}}\right|_{\bar{z}=1} \text { at } \quad \bar{z}_{p}=0 \\
& k_{p} \frac{\partial \bar{T}_{p}}{\partial \bar{x}_{p}}=h_{c} l\left(\bar{T}_{p}-\bar{T}_{a}\right) \quad \text { at } \quad \bar{x}_{p}=0 \\
& -k_{p} \frac{\partial \bar{T}_{p}}{\partial \bar{x}_{p}}=h_{c} l\left(\bar{T}_{p}-\bar{T}_{a}\right) \quad \text { at } \quad \bar{x}_{p}=1 \\
& -k_{p} \frac{\partial \bar{T}_{p}}{\partial \bar{z}_{p}}=h_{c} t_{p}\left(\bar{T}_{p}-\bar{T}_{a}\right) \quad \text { at } \quad \bar{z}_{p}=1
\end{aligned}
$$

The first of these boundary conditions represents continuity of heat flux at the oil-solid interface whereas the second represents convective heat transfer to the environment.

4.3.3 Oil viscosity-temperature and viscositypressure-temperature relationship The energy equation is coupled to Reynolds equation through a nonlinear temperature-viscosity relationship ${ }^{(9)}$ that for most conventional lubricants is:

$$
\bar{\eta}=e^{-\bar{\beta}(\bar{T}-1)}
$$

where: $\bar{\beta}=\beta T_{\text {in }}$ Equation (9.a) is called temperature effect model.

The exponent form of viscosity-temperature and viscosity-pressure relationships are considered as the multiplication effect, which is pointed out by Khonsari and $\operatorname{Wang}^{(10)}$.

$$
\bar{\eta}=e^{-\bar{\beta}(\bar{T}-1)+\bar{\alpha} \bar{p}}
$$

where: $\bar{\alpha}=\frac{\alpha \eta_{0} l u_{b}}{s_{h}^{2}}, \bar{p}=\frac{p s_{h}^{2}}{\eta_{0} l u_{b}}$ Equation (9.b) is called pressure-temperature effect model, which included the temperature and pressure effect.

\section{Calculation of Bearing Performance Parameters}

The pressure distribution can be easily obtained by means of the two-dimensional Reynolds equation based on the pressure boundary conditions and film thickness geometry. Hence, the bearing performance parameters such as normal load carrying capacity, frictional force and number, and side leakage flow can also be calculated.

\subsection{Normal load carrying capacity}

Normal load carrying capacity is the product of pressure distribution and bearing cross-sectional area, which in dimensionless form can be expressed in Eq. (10.a).

$$
\bar{w}_{z}=\frac{w_{z} s_{h}^{2}}{\eta_{0} u_{b} d l^{2}}=\left(\bar{w}_{z x}^{2}+\bar{w}_{z y}^{2}\right)^{\frac{1}{2}}
$$

where: $\bar{w}_{z x}=-\pi \int_{0}^{1} \int_{0}^{1} \bar{p} \cos \bar{\theta} \pi d \bar{\theta} d \bar{x}$

$$
\bar{w}_{z y}=\pi \int_{0}^{1} \int_{0}^{1} \bar{p} \sin \bar{\theta} \pi d \bar{\theta} d \bar{x}
$$




\subsection{Frictional force and number}

Frictional force is the product of the viscous shear stress and bearing cross-sectional area, which in dimensionless form can be expressed as:

$$
\bar{f}=-\pi \int_{0}^{1} \int_{0}^{1}\left(\frac{\bar{h}}{2} \frac{d \bar{p}}{d \bar{x}}+\frac{\bar{\eta}}{\bar{h}}\right) d \bar{\theta} d \bar{x}
$$

Frictional number is the ratio of frictional force to normal load carrying capacity and can be expressed as:

$$
\bar{\mu}=-\frac{\bar{f}}{\bar{w}_{z}}
$$

\subsection{Side leakage flow}

Side leakage flow in the $x$-direction of piston rod velocity can be expressed as:

$$
\bar{q}_{x}=-\frac{\bar{h}^{3}}{12 \bar{\eta}} \frac{\partial \bar{p}}{\partial \bar{x}}+\left(\frac{\bar{h}}{2}\right)
$$

\section{Numerical Method}

Equations (2.b), (4), and (7) are written in terms of central finite difference forms to yield the pressure and temperature fields, except for the term $\partial T / \partial x$ in Eq. (4) where the backward difference is applied. The central finite difference equations are simultaneously solved by the iterative method combined with a successive overrelaxation factor (the optimal value ranging between 1.5 to $1.8,1.7$ is employed in the computation) to accelerate the pressure field convergence, and an under-relaxation factor (the optimal value ranges between 0.8 and $0.95,0.85$ is employed in the computation) to ensure convergence for the temperature field. The iterative procedure is stopped to give the final pressure or temperature distribution when the error in computing the pressure and the temperature in the next iterative procedure is less than $10^{-5}$.

\section{Results and discussion}

Figure 1 shows that the dual conical-cylindrical bearings is studied herein where length $l=45 \mathrm{~mm}$, piston rod diameter $d=45 \mathrm{~mm}$, shoulder-length ratio $n_{s}=0.8$ and outlet film thickness ratio $\bar{h}_{0}$. The bearing performance in terms of pressure distribution $(\bar{P})$, normal load carrying capacity $\left(\bar{w}_{z}\right)$, frictional number $(\bar{\mu})$ and side leakage flow $\left(\bar{q}_{x}\right)$ are also analyzed by the numerical method under piston rod velocity $u_{b}=4 \mathrm{~m} / \mathrm{s}$. Also, the viscosity-

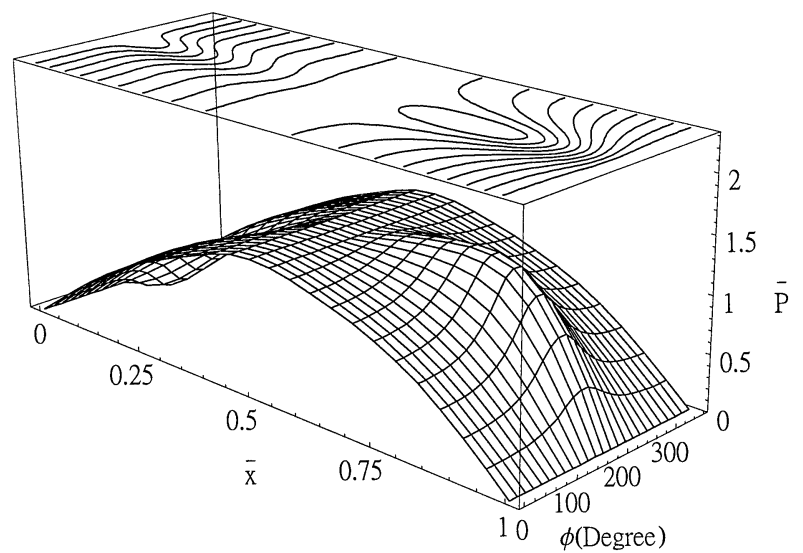

(b) 3-D Pressure distribution for $\left(\bar{h}_{0}=1.2, \bar{e}=0.6, \sigma_{1}=0.1, \sigma_{2}=0.05\right)$

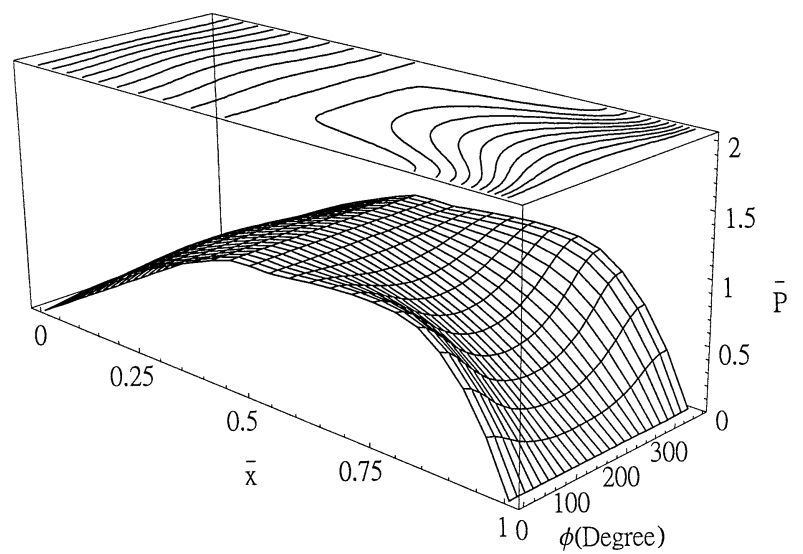

(d) 3-D Pressure distribution for $\left(\bar{h}_{0}=1.2, \bar{e}=0.1, \sigma_{1}=0.6, \sigma_{2}=0.05\right)$ (c) 3-D Pressure distribution for $\left(\bar{h}_{0}=0.6, \bar{e}=0.1, \sigma_{1}=0.6, \sigma_{2}=0.05\right)$

Fig. 4 Pressure distribution 
Table 1 Parameters applicable to results of Figs. 4-8

\begin{tabular}{l|l}
\hline Piston rod diameter & $d=45 \mathrm{~mm}$ \\
Axial length & $l=45 \mathrm{~mm}$ \\
Shoulder length ratio & $n_{s}=0.8$ \\
Radial thickness & $t_{p}=10 \mathrm{~mm}$ \\
Shoulder height & $s_{h}=20 \mu \mathrm{m}$ \\
Supply pressure & $p_{s}=21 \mathrm{MPa}$ \\
Lubricant density at $40^{\circ} \mathrm{C}$ & $\rho_{0}=876 \mathrm{~kg} / \mathrm{m}^{3}$ \\
$\quad$ & \\
Lubricant viscosity at $40^{\circ} \mathrm{C}$ & $\eta_{0}=0.0394 \mathrm{~Pa} \cdot \mathrm{s}\left(\right.$ or N$\left.\cdot \mathrm{s} / \mathrm{m}^{2}\right)$ \\
Lubricant specific heat & $c_{0}=1880 \mathrm{~J} / \mathrm{kg} \mathrm{K}$ \\
Lubricant thermal conductivity & $k_{0}=0.13 \mathrm{~W} / \mathrm{m} \mathrm{K}$ \\
Viscosity-pressure coefficient ${ }^{(9)}$ & $\alpha=1.81 \times 10^{-8} \mathrm{~m}^{2} / \mathrm{N}\left(40^{\circ} \mathrm{C}\right)$ \\
& $\alpha=1.37 \times 10^{-8} \mathrm{~m}^{2} / \mathrm{N}\left(100^{\circ} \mathrm{C}\right)$ \\
& $\alpha=1.13 \times 10^{-8} \mathrm{~m}^{2} / \mathrm{N}\left(150^{\circ} \mathrm{C}\right)$ \\
Temperature viscosity coefficient & $\beta=0.03174 / \mathrm{K}$ \\
Solid thermal conductivity & $k_{p}=48 \mathrm{~W} / \mathrm{m} \mathrm{K}^{2}$ \\
Solid convection heat transfer coefficient & $h_{c}=175 \mathrm{~W} / \mathrm{m}^{2} \mathrm{~K}$ \\
Inlet lubricant temperature & $T_{i n}=40^{\circ} \mathrm{C}$ \\
Ambient temperature & $T_{a}=25^{\circ} \mathrm{C}$ \\
\hline
\end{tabular}

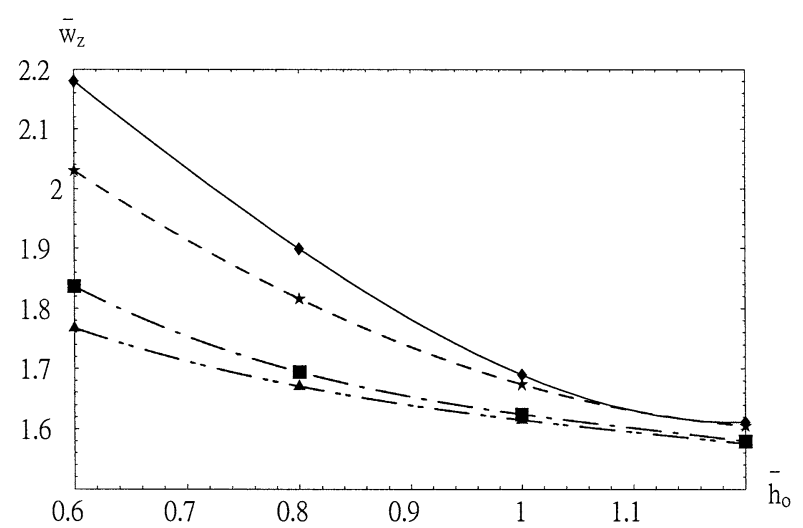

Fig. 5 Normal load carrying capacity as a function of film thickness:

(一): $\left(\bar{e}=0.1, \sigma_{1}=0.6, \sigma_{2}=0.05\right)$, pressure-temperature effect model;

$(\cdots):\left(\bar{e}=0.1, \sigma_{1}=0.6, \sigma_{2}=0.05\right)$, temperature effect model;

$(---): \quad\left(\bar{e}=0.6, \quad \sigma_{1}=0.1, \quad \sigma_{2}=0.05\right)$, pressuretemperature effect model;

$(-\cdot-):\left(\bar{e}=0.6, \sigma_{1}=0.1, \sigma_{2}=0.05\right)$, temperature effect model

$\left(\bar{w}_{z}=1\right.$, i.e., $\left.w_{z}=35.9 \mathrm{kN}\right)$

pressure-temperature variations and thermohydrodynamic temperature boundary conditions are considered herein.

The results presented in Figs. 4-8 were obtained for the inputs listed in Table 1.

Figure 4 (a) illustrates the 3-D pressure distribution for the condition of $\left(\bar{h}_{0}=0.6, \bar{e}=0.6, \sigma_{1}=0.1, \sigma_{2}=0.05\right)$. It indication the maximum pressure existed at the section $\phi=180^{\circ}, \bar{p}_{\max }$ was 2.5 . Figure 4 (b) shows the pressure distribution for the condition of $\left(\bar{h}_{0}=1.2, \bar{e}=0.6, \sigma_{1}=0.1\right.$, $\left.\sigma_{2}=0.05\right)$. The maximum pressure existed at the section $\phi=180^{\circ}$, but maximum pressure is decreased, $\bar{p}_{\text {max }}$ was 2.0. Figure 4 (c) demonstrates the 3 -D pressure distribution for the condition of $\left(\bar{h}_{0}=0.6, \bar{e}=0.1, \sigma_{1}=0.6\right.$, $\left.\sigma_{2}=0.05\right)$. It indication the maximum pressure existed

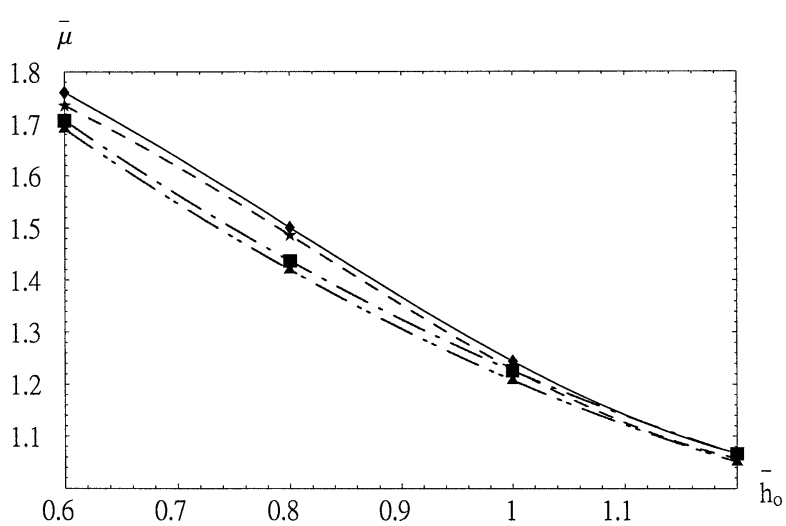

Fig. 6 Frictional number as a function of film thickness: $\left(\right.$ ( ) $:\left(\bar{e}=0.1, \sigma_{1}=0.6, \sigma_{2}=0.05\right)$, pressure-temperature effect model;

$(\cdots):\left(\bar{e}=0.1, \sigma_{1}=0.6, \sigma_{2}=0.05\right)$, temperature effect model;

$(-\cdot): \quad\left(\bar{e}=0.6, \quad \sigma_{1}=0.1, \quad \sigma_{2}=0.05\right)$, pressuretemperature effect model;

$(-\cdot-):\left(\bar{e}=0.6, \sigma_{1}=0.1, \sigma_{2}=0.05\right)$, temperature effect model

$\left(\bar{\mu}=1\right.$, i.e., $\left.\mu=4.4 \times 10^{-4}\right)$

at the section $\phi=0^{\circ}, \bar{p}_{\max }$ was 6.0. Figure $4(\mathrm{~d})$ shows the 3-D pressure distribution for the condition of $\left(\bar{h}_{0}=1.2\right.$, $\left.\bar{e}=0.1, \sigma_{1}=0.6, \sigma_{2}=0.05\right)$. The maximum pressure existed at the section $\phi=0^{\circ}$, but maximum pressure is decreased, $\bar{p}_{\max }$ was 1.5 . That an increase film thickness $\left(\bar{h}_{0}\right)$ results in decreased the pressure distribution.

Figure 5 displays the variation in normal load carrying capacity versus the film thickness, pressure-thermal effect model and thermal effect model. As seen in the Fig. 5, the normal load carrying capacity $\bar{w}_{z}$ decreased while the film thickness $\left(\bar{h}_{0}\right)$ increased under the same conditions. Also, $\bar{w}_{z}$ with pressure-temperature effect model is higher than that with the temperature effect model, and the conditions of ( $\left.\bar{e}=0.1, \sigma_{1}=0.6, \sigma_{2}=0.05\right)$ is higher than with the conditions of $\left(\bar{e}=0.6, \sigma_{1}=0.1\right.$, $\left.\sigma_{2}=0.05\right)$.

Figure 6 illustrates the variation of the frictional number versus the film thickness for $\left(\bar{e}=0.1, \sigma_{1}=0.6, \sigma_{2}=\right.$ $0.05)$ and $\left(\bar{e}=0.6, \sigma_{1}=0.1, \sigma_{2}=0.05\right)$. According to the Fig. 6 results, the frictional number $(\bar{\mu})$ decreases with an increase of film thickness $\left(\bar{h}_{0}\right)$.

Figure 7 illustrates the variation of the side leakage flow versus the film thickness for $\left(\bar{e}=0.1, \sigma_{1}=0.6, \sigma_{2}=\right.$ $0.05)$ and $\left(\bar{e}=0.6, \sigma_{1}=0.1, \sigma_{2}=0.05\right)$. According to the Fig. 7 results, the side leakage flow $\left(\bar{q}_{x}\right)$ increases with an increase of the film thickness $\left(\bar{h}_{0}\right)$.

Figure 8 (a) shows the distribution, which distributes alone $\bar{\theta}$ direction, of maximum temperature of oil film inner under conditions of $\left(\bar{e}=0.6, \sigma_{1}=0.1, \sigma_{2}=0.05\right)$ and the maximum temperature existed at the film exit above. From Fig. $8(\mathrm{a})$, it is obvious that the film thickness $\left(\bar{h}_{0}\right)$ 


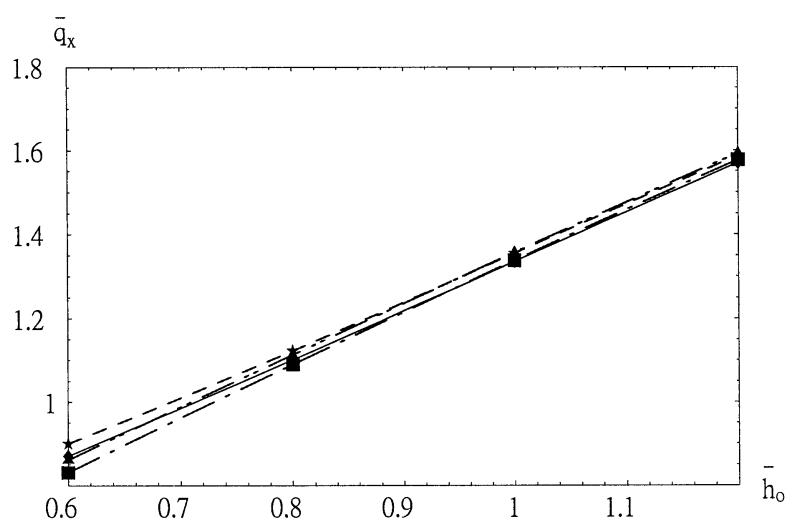

Fig. 7 Side leakage flow as a function of film thickness: $(-):\left(\bar{e}=0.1, \sigma_{1}=0.6, \sigma_{2}=0.05\right)$, pressure-temperature effect model;

$(\cdots):\left(\bar{e}=0.1, \sigma_{1}=0.6, \sigma_{2}=0.05\right)$, temperature effect model;

$(-\cdot): \quad\left(\bar{e}=0.6, \quad \sigma_{1}=0.1, \quad \sigma_{2}=0.05\right)$, pressuretemperature effect model;

$(-\cdot-):\left(\bar{e}=0.6, \sigma_{1}=0.1, \sigma_{2}=0.05\right)$, temperature effect model

$\left(\bar{q}_{x}=1\right.$, i.e., $\left.q_{x}=3.6 \mathrm{~cm}^{3} / \mathrm{s}\right)$

increased, $\bar{T}_{\max }$ also increases, and the maximum temperature existed at the section $\phi=0^{\circ}$. Furthermore, the maximum temperature distribution in pressure-temperature effect model is higher than that in temperature effect model.

Figure 8 (b) shows the value of maximum temperature, which distributes alone $\bar{\theta}$ direction, of oil film inner under conditions of $\left(\bar{e}=0.1, \sigma_{1}=0.6, \sigma_{2}=0.05\right)$ and the maximum temperature existed at the film exit above. According to the Fig. 8 (b), the maximum temperature existed at the section $\phi=180^{\circ}$ under the film thickness $\bar{h}_{0}=1.2$. But the maximum temperature existed at the section $\phi=0^{\circ}$ under the film thickness $\bar{h}_{0}=0.6$.

\section{Conclusions}

In this study, the performance parameters of hydraulic servo cylinder using dual conical-cylindrical bearing have been analyzed herein via dimensionless variables by numerical methods. Based on those results, we concluded as follows:

1. The pressure effect increases viscosity distribution in the oil film. Interactions occur among pressure, viscosity and temperature. The effect of viscositypressure is more conspicuous than that of viscositytemperature. Moreover, the normal load carrying capacity in the pressure-thermal effect model is higher than that in the thermal effect model.

2. Both the normal load carrying capacity and frictional number increase with decreasing film thickness, but the side leakage flow decreases with decreasing film thickness.

3. The design value of film thickness is necessary to consider the internal diameter of bearing machining toler-

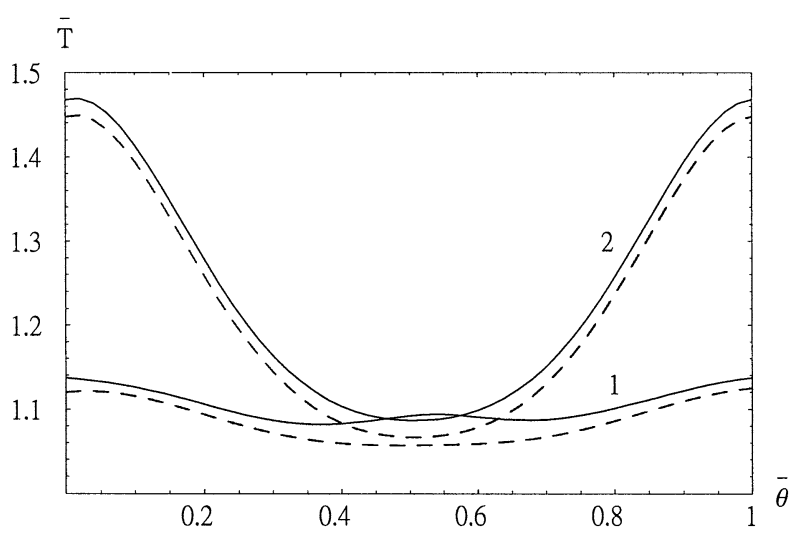

(a) Temperature distribution in the inner film section under the conditions:

1: $\left(\bar{h}_{0}=0.6 ; \bar{e}=0.6, \sigma_{1}=0.1, \sigma_{2}=0.05\right)$;

$2:\left(\bar{h}_{0}=1.2 ; \bar{e}=0.6, \sigma_{1}=0.1, \sigma_{2}=0.05\right)$;

(一): pressure-temperature effect model;

$(\cdots)$ : temperature effect model;

$\left(\bar{T}=1\right.$, i.e., $\left.T=40^{\circ} \mathrm{C}\right)$

$\overline{\mathrm{T}}$

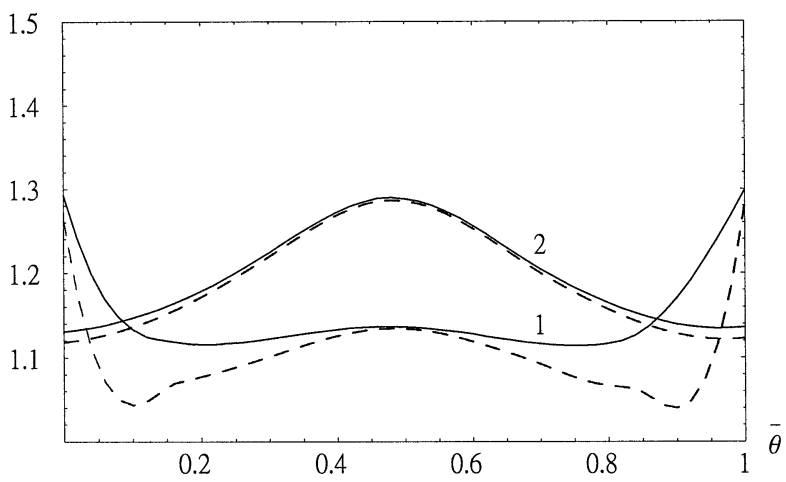

(b) Temperature distribution in the inner film section under the conditions:

1: $\left(\bar{h}_{0}=0.6 ; \bar{e}=0.1, \sigma_{1}=0.6, \sigma_{2}=0.05\right)$;

$2:\left(\bar{h}_{0}=1.2 ; \bar{e}=0.1, \sigma_{1}=0.6, \sigma_{2}=0.05\right)$;

$(-)$ : pressure-temperature effect model;

$(\cdots)$ : temperature effect model;

$\left(\bar{T}=1\right.$, i.e., $\left.T=40^{\circ} \mathrm{C}\right)$

Fig. 8 Maximum temperature as a function of circumferential angle

ance, real roundness and straightness. Therefore, the best scope of $\bar{h}_{0}$ is taken as $0.8-1.0$.

\section{Acknowledgment}

The authors would like to thank the National Science Council of the Republic of China, Taiwan for financially supporting this research under Contract No. NSC92-2212E-159-005.

\section{References}

( 1 ) Yang, Y.K. and Jeng, M.C., Analysis of Thermal Effects on the Misaligned Hydraulic Servo Cylinder, Trib. Int., Vol.34 (2001), pp.95-106.

( 2 ) Jeng, M.C. and Yang, Y.K., Comparison of Thermal Effects on the Conical-Cylindrical Bearing with 2-D 
and 3-D Energy Equations, Trib. Trans., Vol.45 (2002), pp.67-75.

( 3 ) Yang, Y.K. and Jeng, M.C., Thermohydrodynamic Analysis of the Misaligned Conical-Cylindrical Bearing with Non-Newtonian Lubricants, Trib. Trans., Vol.46 (2003), pp.161-169.

(4) Yang, Y.K. and Jeng, M.C., Analysis of Viscosity Interaction and Heat Transfer on the Dual ConicalCylindrical Bearing, Trib. Trans., Vol.47 (2004), pp.77-85.

( 5 ) Buckholz, R.H. and Lin, J.F., The Effect of Journal Bearing Misalignment on Load and Cavitation for Non-Newtonian Lubricants, ASME Jour. of Trib., Vol.108 (1986), pp.645-654.

( 6 ) Jang, J.Y. and Chang, C.C., Adiabatic Solution for a Misaligned Journal Bearing with Non-Newtonian Lu- bricants, Trib. Int., Vol.20, No.5 (1987), pp.267-274.

( 7 ) Ezzat, H.A. and Rohde, S.M., A Study of the Thermohydrodynamic Performance of Finite Slider Bearings, ASME Jour. of Lubr. Tech., Vol.95 (1973), pp.298307.

( 8 ) Jang, J.Y. and Khonsari, M.M., Thermohydrodynamic Design Charts for Slider Bearing, ASME Jour. of Trib., Vol.119 (1997), pp.733-740.

(9) Hamrock, B.J., Fundamentals of Fluid Film Lubrication, (International Editions), (1994), pp.57-67, McGraw-Hill Book Co., Singapore.

(10) Khonsari, M.M. and Wang, S.H., On the FluidSolid Interaction in Reference to Thermoelastohydrodynamic Analysis of Journal Bearings, ASME Jour. of Trib., Vol.119 (1991), pp.398-403. 